\title{
Magnetic field sensor based on multi-port microcoil resonator
}

\author{
W. Talataisong*, ${ }^{*}$, R. Ismaeel ${ }^{\dagger}$, A. Masoudi, M. Beresna and G. Brambilla \\ Optoelectronics Research Centre, University of Southampton, Southampton, SO17 1BJ, UK \\ †These authors contributed equally \\ *Corresponding author:w.talataisong@ soton.ac.uk
}

\begin{abstract}
A multi-port microcoil resonator magnetic field sensor based on a microfiber coupler coil resonator (MMCR) is presented. The microfiber coupler coil is fabricated by coiling a four-port microfiber coupler with a uniform waist region around a low index support rod. The MMCR is embedded in a low refractive index polymer to increase the robustness and operation stability. The enhanced sensor response to the magnetic field is ascribed to the diverse MMCR response to the light polarization state. The MMCR magnetic field sensor is compact and low cost, and exhibits a magnetic field sensitivity of $37.09 \mathrm{~dB} / \mathrm{T}$ with an estimated minimum detection limit (DL) of $\sim 27 \mu \mathrm{T}$.
\end{abstract}

Keywords: Magnetic field sensor; Multi-port microcoil resonator; Microfiber coupler; Faraday effect

\section{INTRODUCTION}

Currently, magnetic field sensors are widely used in many applications such as navigation, environment monitoring, aerospace and smart grids. Magnetic field sensors based on optical fibers have attracted a great deal of attention owing to their advantageous features such as good electric insulation, large dynamic range, and compactness [1]. Many optical fiber magnetic field sensors have been reported, especially based on magnetic fluids [2-5]. Yet, the low durability and the large size of this type of sensors are their main drawbacks. Recently, the advances in optical microfibers resulted in the development of numerous sensors based on microcoil resonators (MCR) [3,5].

Here, we present a novel magnetic field sensor based on a multi-port microcoil resonator (MMCR). The main advantage of the magnetic field sensor is its high polarization sensitivity. An asymmetric change in the intensity of the transmission spectrum $[6,7]$ is used to analyse the magnetic field sensitivity of the device.

\section{PRINCIPLE OF OPERATION}

A microfiber coupler (MC) is an optical fiber device which is composed of two conical transition regions and a central uniform waist region [8] with a diameter in the order of few microns where the coupling occurs. A multi-port microcoil resonator (MMCR) is based on a microfiber coupler wrapped around a low index support rod [9], thus it benefits from the advantages of both the resonator and the coupler. Important features of an MMCR include the physical separation between the output ports and the high polarization dependent extinction ratio. The Faraday effect describes the interaction between light and magnetic field in a medium. The magnetic field causes a rotation in the polarization azimuth $\left(\theta_{0}\right)$ of linearly polarized light which is proportional to the medium Verdet constant $(V)$ and the length of interaction. In a microcoil resonator, the Faraday rotation associated with a single turn is given by: $[6,7]$

$$
\theta_{0}=V B(2 \pi r)
$$

where $B$ is the magnetic field and $r$ is the MMCR radius. While silica exhibits an exceedingly small Verdet constant, in this sensing system the overall Faraday rotation is enhanced by the number of turns in the microfiber coupler coils and by the resonating effect of the coil which effectively increases the light optical path. The MMCR exhibits an extremely large polarization dependence: the two orthogonal polarizations have significantly different resonance spectra, both in terms of resonance wavelength and strength $[6,7]$. Thus, any changes in the azimuth of the light polarization results in a shift and a change of the extinction ratio of the resonances, and therefore, in the overall MMCR transmission spectrum. By monitoring the changes in the MMCR transmission properties at different wavelengths, it is possible to retrieve the fraction of mode in each polarization, thus $\theta_{0}$ and $B$. Here the wavelengths are chosen to maximize this effect. 


\section{DEVICE FABRICATION AND EXPERIMENTAL SETUP}

A schematic of the MMCR is presented in figure 1. The MMCR was assembled by using a microfiber coupler (MC) fabricated from two standard telecom fibers (Corning SMF-28) using the modified flame brushing technique [10]. The two single mode fibers were stripped of their acrylic coating over a length of $\sim 100 \mathrm{~mm}$ and twisted 1.5 turns around each other. The twisted region was fused together using a ceramic micro-heater with an estimated temperature of $\sim 1450{ }^{\circ} \mathrm{C}$. In the experiment, the transition regions of the $\mathrm{MC}$ were $\sim 3 \mathrm{~cm}$ and the length of the uniform waist region was $4 \mathrm{~mm}$ with a waist diameter of $\sim 3.2 \mu \mathrm{m}$. To create the MMCR, the fabricated microfiber coupler was wrapped 3 times around a $1 \mathrm{~mm}$ diameter glass rod coated with Teflon. The coiled microfiber coupler was embedded in a low refractive index polymer matrix which was cured to enhance its durability. An optical microscope image of the coiled MC with the $\sim 3.5 \mu \mathrm{m}$ spacing between the adjacent loops is shown in figure 2(a).

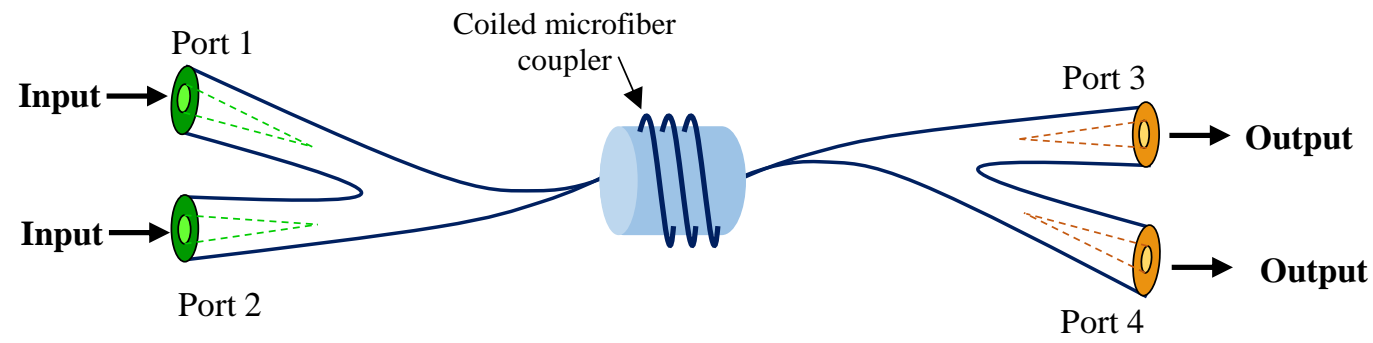

Figure 1. Schematic of the multi-port microcoil resonator (MMCR) fabricated by coiling the micro fiber coupler 3-turns around a low-refractive rod coated with Teflon.

Figure 2(b) illustrates the Helmholtz coils used to generate the magnetic field. The magnetic field was enhanced by placing two iron rods into the Helmholtz coils. The Helmholtz coils were connected to a power supply to generate magnetic field as a result of the current flowing into the coils. The strength of magnetic field was adjusted by tuning the magnitude of the applied current. In the experimental setup, the multi-port microcoil resonator was located between two iron rods. Light from an incoherent white light source (Bentham, WLS100) was launched into port 1 of the MMCR and the output of port 3 was connected to an optical spectrum analyzer (Yokogawa, AQ6370) to monitor the transmission spectrum. The strength of the magnetic field was measured by a gauss meter (Hirst, GM07) and related to the applied current to create the calibration curve.
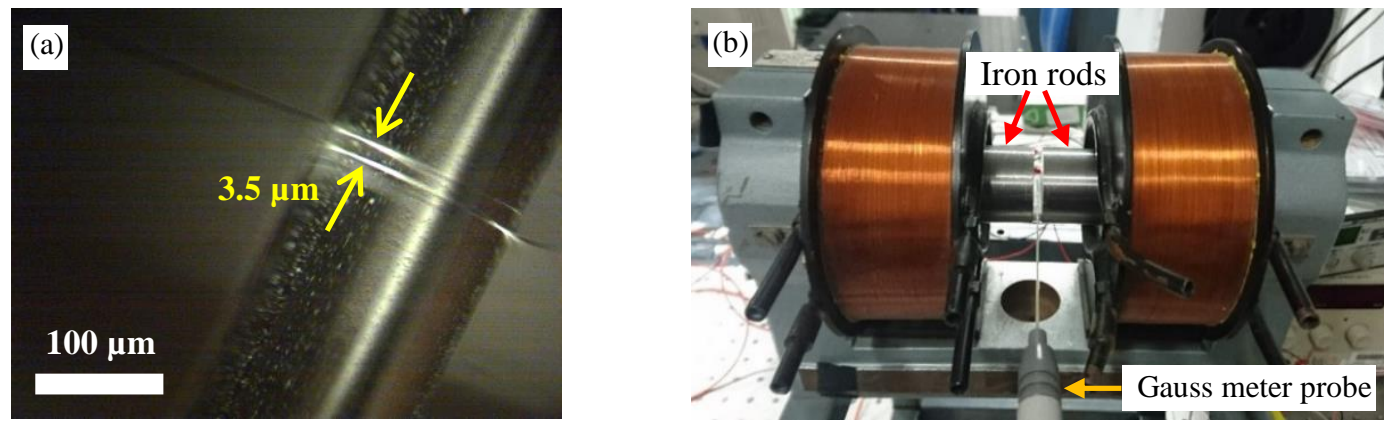

Figure 2. (a) Microscope image of the optical fiber coupler wrapped around a low refractive-index support rod. The distance between adjacent turns of the coil is $\sim 3.5 \mu \mathrm{m}$. (b) Helmholtz coils and iron rods used to generate the uniform magnetic field with the maximum strength of $1.2 \mathrm{~T}$.

\section{RESULTS AND DISCUSSION}

In the magnetic field measurements, currents ranging from $0 \mathrm{~A}$ to $0.8 \mathrm{~A}$ were applied to the coils to generate the magnetic field with strengths ranging from $0 \mathrm{~T}$ to $0.517 \mathrm{~T}$. The transmission spectrum of the MMCR was monitored in real-time using the OSA. Figure 3(a) shows the transmission spectra for magnetic field strengths of 0, 0.129, 0.259 , $0.323,0.389$, and $0.517 \mathrm{~T}$, respectively. The raw data in figure 3(a) reveals the random changes in the amplitude of the 
spectra caused by noise. The amplitude noise was reduced by smoothing the acquired data with a 30-point averaging. This effectively corresponds to using a powermeter and a passband filter with an aperture corresponding to 10pm. The plot of the smoothed transmission spectra is illustrated in figure 3(b). The result shows that the noise is greatly reduced while the dips are hardly changed. A red shift of the transmission spectra can be observed with increasing the magnetic field strength. Figure 3(c) presents the resonance wavelength shift as a function of the magnetic field strength. The data shows a sinusoidal relationship between the resonance wavelength and the magnetic field strength with an $\mathrm{R}^{2}$ value of 0.996 . The magnetic field sensitivity of the sensor was measured to be $65.07 \mathrm{pm} / \mathrm{T}$.
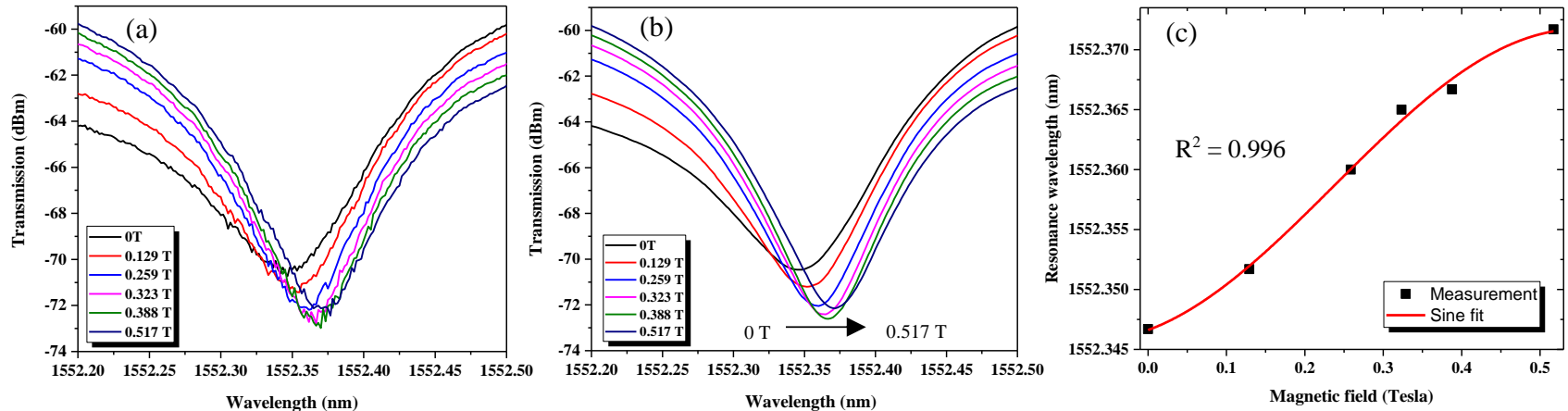

Figure 3. (a) Raw and (b) smoothed transmission spectra of the multi-port microcoil resonator (MMCR) under different magnetic field strengths. Smoothing used an average of 30 points. (c) Dependence of the resonance wavelength on the magnetic field strength. The graph slope provides the sensor sensitivity $(65.07 \mathrm{pm} / \mathrm{T})$.

Spectra along larger wavelength ranges were recorded and are shown in figure 4(a). Unlike the small shift of the resonance wavelength associated to increasing magnetic fields, the variation in the intensity of the transmission spectra at different wavelengths for different magnetic field strengths can be clearly observed and it was ascribed to the strong polarization dependence of the Faraday effect in MMCRs [6, 7]. In figure 4(a) the recorded power changes at the wavelengths $\lambda_{1}(\lambda=1552.73 \mathrm{~nm})$ and $\lambda_{2}(\lambda=1552.837 \mathrm{~nm})$ for different magnetic field strengths provide an alternative detection mechanism for magnetic field sensing. While the transmission at the wavelength $\lambda_{1}$ decreases with the increasing magnetic field strengths, it increases at the wavelength $\lambda_{2}$. The dependence of the fiber output at the wavelengths $\lambda_{1}$ and $\lambda_{2}$ on the magnetic field strength is summarized in figures 4(b) and 4(c), respectively, and show a complementary behavior and some degree of noise. This complementarity was exploited in a balanced detection to eliminate the noise associated to the intensity fluctuations of the light source. Figure 5 shows the relative change in spectral density at the two wavelengths $\lambda_{1}$ and $\lambda_{2}\left(\left[\left(\mathrm{P}_{1}(\mathrm{H}=0)-\mathrm{P}_{1}(\mathrm{H})\right]-\left[\left(\mathrm{P}_{2}(\mathrm{H}=0)-\mathrm{P}_{2}(\mathrm{H})\right]\right)\right.\right.$ as a function of the applied magnetic field. By fitting a sinusoidal function to the data points, the slope at the origin was calculated to be equal 37.09 $\mathrm{dB} / \mathrm{T}$, providing an overall magnetic field sensitivity of $37.09 \mathrm{~dB} / \mathrm{T}$ in the magnetic field range of $0 \sim 0.517 \mathrm{~T}$. A minimum detection limit (DL) of $\sim 2.70 \times 10^{-5} \mathrm{~T}$ was estimated.
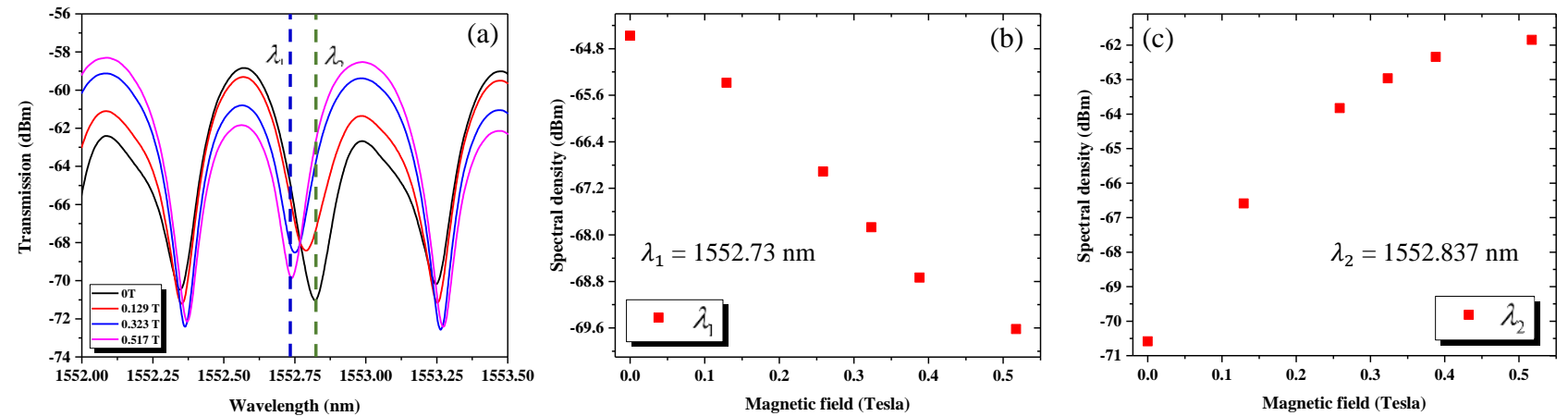

Figure 4. (a) Change in the MMCR transmission at different magnetic field strengths. (b) Changes in the spectral density at the wavelength $\lambda_{1}(\lambda=1552.73 \mathrm{~nm})$ for different magnetic field strength. (c) Changes in the spectral density at the wavelength $\lambda_{2}(\lambda=1552.837 \mathrm{~nm})$ for different magnetic field strengths. 


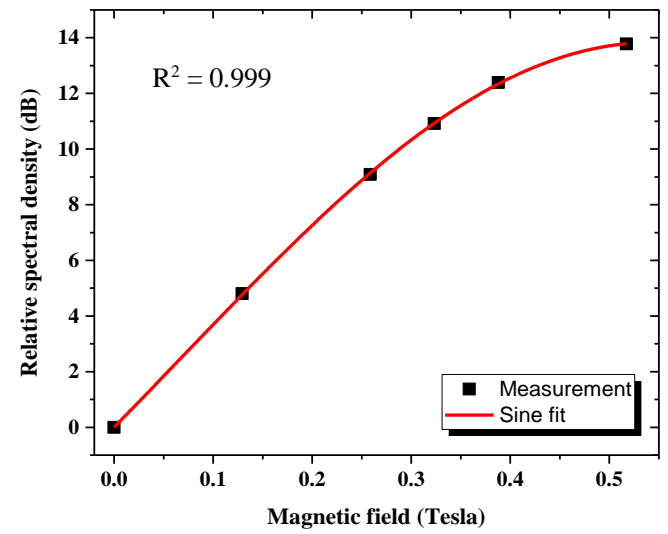

Figure 5. Dependence of the relative spectral density change $\left(\left[\left(\mathrm{P}_{1}(\mathrm{H}=0)-\mathrm{P}_{1}(\mathrm{H})\right]-\left[\left(\mathrm{P}_{2}(\mathrm{H}=0)-\mathrm{P}_{2}(\mathrm{H})\right]\right)\right.\right.$ on the magnetic field.

\section{CONCLUSION}

In conclusion, a novel optical fiber magnetic field sensor based on the Faraday effect in a multi-port microfiber coupler is demonstrated. As the magnetic field rotates the light polarization azimuth in the MMCR, it changes the extent of the resonances associated with the two orthogonal polarizations. This overall results in a shift in the transmission wavelength dips and a change in the intensity of the transmission spectrum. The shift in the wavelength dips were shown to be small in comparison with the changes in the spectrum spectral densities. Thus, the sensitivity of the sensor was measured using the intensity of the asymmetric transmission spectra at selected wavelengths on either side of a resonance. A magnetic field sensitivity of $37.09 \mathrm{~dB} / \mathrm{T}$ was recorded at small magnetic fields, with an estimated detection limit of $\sim 27 \mu \mathrm{T}$.

\section{ACKNOWLEDGEMENT}

One of authors (W. Talataisong) would like to acknowledge the Development and Promotion of Science and Technology Talents Project (DPST) of Thailand for the student scholarship.

\section{REFERENCES}

[1] T. Shen, Y. Feng, B. Sun et al., "Magnetic field sensor using the fiber loop ring-down technique and an etched fiber coated with magnetic fluid," Applied Optics, 55(4), 673-678 (2016).

[2] X. Q. Lei, B. J. Peng, D. R. Chen et al., "An All-Fiber Magnetic Field Sensor Based on Dual-S-Shaped Optic Fiber Integrated With Magnetic Fluid,” IEEE Sensors Journal, 16(4), 958-964 (2016).

[3] X. Li, and H. Ding, "All-fiber magnetic-field sensor based on microfiber knot resonator and magnetic fluid," Optics Letters, 37(24), 5187-5189 (2012).

[4] F. Lv, C. Han, H. Ding et al., "Magnetic Field Sensor Based on Microfiber Sagnac Loop Interferometer and Ferrofluid," IEEE Photonics Technology Letters, 27(22), 2327-2330 (2015).

[5] Y. Zheng, X. Dong, C. C. Chan et al., "Optical fiber magnetic field sensor based on magnetic fluid and microfiber mode interferometer," Optics Communications, 336, 5-8 (2015).

[6] G. Y. Chen, T. Lee, R. Ismaeel et al., "Resonantly Enhanced Faraday Rotation in an Microcoil Current Sensor," IEEE Photonics Technology Letters, 24(10), 860-862 (2012).

[7] G. W. Day, D. N. Payne, A. J. Barlow et al., "Faraday rotation in coiled, monomode optical fibers: isolators, filters, and magnetic sensors," Optics Letters, 7(5), 238-240 (1982).

[8] Y. Jung, G. Brambilla, and D. J. Richardson, "Optical microfiber coupler for broadband single-mode operation," Optics Express, 17(7), 5273-5278 (2009).

[9] R. Ismaeel, T. Lee, F. Al-Saab et al., "A self-coupling multi-port microcoil resonator," Optics Express, 20(8), 8568-8574 (2012).

[10] G. Brambilla, "Optical fibre nanowires and microwires: a review,” Journal of Optics, 12(4), 043001 (2010). 